\title{
Author Index of Volume 218 Issues 1-4
}

Abu-Surrah, A. S., 537

Aghabozorg, H., 75, 432

Ahlgren, M., 537

Ahmed, M., 90

Akriche, S., 143

Akselrud, L. G., 414, 415

Al-Shami, E. M., 537

Altin, E., 541

Àlvarez Micó, X., 547, 549

Amort, Ch., 187

Arif, A. M., 47, 49

Arslan, H., 479

Aslantaş, M., 175

Avila, R., 35

Babizhetskyy, V., 173, 417

Bagatin, I. A., 427

Balegroune, F., 427

Banerji, A., 337

Barberan, O., 123

Barbier, B., 9, 265, 405, 409

Basta, R., 49

Bauer, A., 473

Bauer, J., 173

Baxla, A. P., 57

Bayot, D., 477, 572

Baysen, F., 175

Beck, W., 113

Bekaert, A., 123

Beketov, K. M., 203

Ben Salah, A., 29, 399

Bianchi, L., 219

Bierer, L., 107

Biesemeier, F., 164, 419

Bijanzadeh, H. R., 189

Bildstein, B., 184, 187

Binnemans, K., 118, 197, 488

Binnewies, M., 373

Bittner, S., 441

Blanton, T. N., 439

Böhmer, V., 429

Borrmann, H., 17, 167, 169, 171

Bradshaw, J. S., 569

Braun, N. A., 471

Bringmann, G., 209

Brion, J. D., 123

Bruhn, C., 115

Brunner, H., 125

Budnyk, S., 161

Buhl, J.-Ch., 275

Bujici, T. M., 148

Burns, M., 347

Çakır, B., 175

Capparelli, M. V., 35

Cardoso Gil, R., 397

Carrillo-Cabrera, W., 397

Casellato, U., 343, 437

Cassels, B. K., 77, 79, 81, 83, 177

Castedo, L., 77, 79, 81, 83, 177

Cérésiat, M., 145

Chaichit, N., 105

Champarnaud-Mesjard, J.-C., 293

Charris, J. E., 35

Chen, K., 225

Chen, S.-H., 301

Cheng, Y.-Q., 95

Ciufolini, M. A., 39

Coghlan, D. R., 359, 361

Cowley, A., 31

Cvek, B., 205

Dalley, N. K., 569
Danan, A., 123

Dehaen, W. 485

Deiseroth, H. J., 5, 6

Députier, S., 173

Devillers, I., 194

Devillers, M., 59, 477, 572

Dey, S., 52, 55, 57, 179

Doering, Th., 391, 393, 395

Domínguez, J. N., 35

Dorhout, P. K., 19, 20

Duliere, E., 59

Easton, C. J., 359, 361, 363, 365

Ehlinger, N., 87

Ehrenberg, H., 29

El-Saddek, M., 90

Elwahy, A. H. M., 90

Emen, F., 479

Engering, J., 153, 199, 201

Ernst, R. D., 47, 49

Ettorre, R., 343

Euler, H., 9, 265, 405, 409, 471, 473

Ewald, B., 377

Fang, Q., 323, 325, 545

Fang, Y.-W., 221

Fässler, T. F., 370, 455, 458, 461

Feng, X.-C., 271

Feng, Y.-L., 239, 241, 527, 529, 531, 533

Fornasini, M. L., 279

Fossa, P., 219

Frey, W., 107, 109

Fruziński, A., 191

Fu, Y.-S., 339

Fuess, H., 29, 90, 269

Fun, H.-K., 261, 317, 511, 521

Galdámez, A., 151, 403

Garbarczyk, J., 567

Garland, M. T., 151, 403

Ge, M.-H., 165, 273

Geist, V., 391, 393, 395

Gesing, Th. M., 275

Gesland, J.-Y., 3

Goel, V. K., 55, 57

Gray, A. K., 19, 20

Graziani, R., 343, 437

Greatrex, B. W., 63, 65, 561

Grin, Yu., 159, 161, 397

Grosvallet, L., 39

Grzechnik, A., 3

Gu, J.-M., 497

Guérin, R., 173

Guo, Y.-W., 328

Haase, D., 101

Haase, W., 90

Hanfland, M., 414, 415

Harms, K., 164, 419

Harringer, N. A., 287

Harvey, B. G., 47

Hauptmann, R., 370, 455, 458, 461

He, H.-Y., 245, 563

He, M.-Y., 21

Held, P., 13, 15

Hillebrand, G., 464, 467

Hirt, H., 5, 6

Ho, S. Y., 73

Hodali, H. A., 537

Höhn, P., 163

Hu, M.-L., 93, 95, 367, 491, 494, 565

Hu, X.-R., 497

Hu, Z. G., 1

Huang, H., 443
Huang, H.-P., 443

Huang, Y.-X., 17, 165, 167, 169, 171, 273

Hughes, C. M. M., 363, 365

Ibragimov, B. T., 203

Illos, R. A., 441

Itoh, K., 333

Jäger, V., 107, 109

Jaiboon-Muangsin, N., 105

Jain, V. K., 67, 69, 71

Jansen, M., 153, 199, 201, 379, 381

Jardin, R., 173

Jäschke, B., 381

Jiang, J.-Y., 229

Jiang, M.-H., 543

Jiménez, M., 151

Jin, Q.-H., 45, 443

Joswig, W., 429

Kabadou, A., 29, 269, 399

Kai, Y., 1

Kaloun, E. B., 123

Kameníček, J., 205

Kanehisa, N., 1

Karolak-Wojciechowska, J., 191, 331

Karray, R., 399

Kempe, R., 99, 464, 467

Kendi, E., 175

Khedhiri, L., 233

Khorphueng, P., 105

Kimber, M. C., 63, 65

Kirchmaier, R., 541

Kirfel, A., 9, 265, 405, 409, 471, 473

Kleinpeter, E., 217

Klepp, K. O., 287, 383, 385, 387, 539

Klinga, M., 537

Klubek, K. P., 439

Kniep, R., 17, 165, 167, 169, 171,273,377

Kochel, A., 207, 235

Köhler, J., 276

Kolitsch, U., 401

Köllnberger, A., 125

Kong, Z.-P., 223, 225

Konkol, M., 115

Kopacka, H., 184

Kovacs, J., 217

Kowalska, T., 191

Kowalski, P., 191

Kozioł, A. E., 37

Krilow, M., 429

Külcü, N., 479

Kumar, P., 52, 179

Kutoglu, A., 7

Kuz'ma, Yu. B., 159, 161

Lamartine, R., 87

Lamb, J. D., 569

Lange, J., 331

Laurent, M., 145

Lemoine, P., 123

Lentz, A., 541

Leroi, C., 39

Li, S.-H., 443

Li, S.-J., 11,148

Li, S.-Y., 43, 319

Li, X., 45, 211, 213, 445, 448, 451, 453

Li, X.-H., 367, 491, 494

Li, Y., 521

Li, Y.-H., 41, 319

Lin, H., 525, 533

Lin, J.-L., 227, 229

Lin, Q.-Y., 531

Lis, T., 37 
Liu, C.-Q., 297

Liu, G.-F., 263

Liu, G.-Q., 545

Liu, H.-L., 41, 319

Liu, L.-H., 339

Liu, Q.-X., 259, 261, 317

Liu, W.-J., 255, 311

Liu, W.-S., 519

Liu, X., 259, 313, 315, 335

Liu, X.-Y., 257

Liu, Y.-Y., 21

Liu, Z., 543

Lobring, K. C., 11

Louati, A., 423

Loukil, M., 29, 269, 399

Lu, G.-L., 497

Lu, J.-J., 97

Lv, M.-K., 389

Ma, H.-W., 443

Ma, J.-L., 499, 501, 503, 506, 509, 511, $513,515,517,519,523$

Maccagno, M., 219

Mahalakshmi, H., 67, 69, 71

Mahjoub, A. R., 121, 189, 435

Makhkamov, K. K., 203

Makker, J., 52, 55, 179

Malinka, W., 235

Manfrinetti, P., 279

Manriquez, V., 151, 403

Mao, S.-Y., 17, 171, 271

Maramatsu, K., 1

Marchand-Brynaert, J., 59, 145, 194, 475

Martin, B. R., 19, 20

Martin-Gil, J., 557

Masson, O., 293

Mathews, M. D., 276

Matos Beja, A., 557

Matt, D., 423, 427

Mattausch, Hj., 281, 282, 283, 376, 417

Mayer, P., 113

Mazzone, D., 279

Meents, A., 9, 265, 405, 409

Meier, M., 471

Meiners, F., 101

Meng, F.-J., 253, 261, 263, 321

Meng, L., 97

Mi. J.-X., 17, 165, 167, 169, 171, 271, 273

Mizuguchi, J., 127, 129, 131, 134, 137, 139,141

Moghimi, A., 75, 432

Moors, D., 118

Moretzki, O., 391, 393, 395

Morgenroth, W., 391, 393, 395

Mori, Y., 1

Morsali, A., 33, 121, 189, 237, 299, 435

Moubaraki, B., 345, 347, 349, 351, 354, 357

Mrozek, A., 331

Mugnoli, A., 219

Müller, C. E., 473

Müller, L., 373

Müller, U., 164, 216, 419

Murray, K. S., 345, 347, 349, 351, 354, 357

Nazarenko, A. Y., 569

Nesper, R., 289, 291

Neugebauer, M., 471

Niewa, R., 163

Noguera, O., 293

Noll, A., 216

Nuss, J., 201

Oeckler, O., 281, 282, 283

Önkol, T., 175

Pacco, A., 197

Paixão, J. A., 557

Pal, A., 337
Pan, Y.-J., 43, 253, 301, 303

Paschen, S., 397

Pastorek, R., 205

Pastushok, V. N., 569

Paulus, E. F., 429, 575

Peikert-Regelien, M., 429

Pérez-Lourido, P., 177

Perrin, M., 39

Peters, E.-M., 199, 209, 379

Peters, K., 209

Petrillo, G., 219

Petsom, A., 105

Pfeifer, R.-M., 209

Pogorzelec-Glaser, K., 567

Polamo, M., 553

Pradhan, P., 337

Preishuber-Pflügl, H., 383. 387

Prots, Yu., 159, 161, 377

Pu, S.-Z., 535

Qiu, X.-Y., 499, 501, 503, 506, 509, 511,

$513,515,517,523$

Qu, Y., 519

Rabhi, C., 123

Rajeswaran, M., 439

Ramazani, A., 33, 237, 299, 435

Ramos Silva, M., 557

Ranjbar, M., 75, 432

Redcliffe, J. L., 109

Redzicka, A., 235

Rees, J.-F., 194

Richter, M., 547, 549

Robiette, R., 475

Roengsumran, S., 105

Roger, J., 173

Rong, N.-N., 521

Rubin-Preminger, J. M., 441

Ruiz León, D., 151, 403

Saadioui, M., 429

Saak, W., 101

Sahini, G., 55

Salah, A. B., 269

Sasaki, T., 1

Saunders, A., 31

Schapp, J., 113

Schareina, T., 99

Schilde, U., 217

Schleid, Th., 285

Schmidhammer, H., 181

Schmidt, H., 539

Schütz, J., 181

Schwarz, S., 547, 549

Schwarz, U., 414, 415

Seichter, W., 469

Senju, T., 129

Setsune, J.-I., 333

Shan, Y.-K., 21

Shao, S.-C., 499, 501, 503, 506, 509, 511, 513,517

Shi, H., 328

Shi, H.-Z., 21

Shi, J.-M., 97, 157, 295, 297

Shi, Q., 565

Shi, X.-Z., 389

Shikamori, H., 127

Simon, A., 281, 282, 283, 376, 417

Sing, M., 385

Singh, T. P., 52, 55, 57, 179, 337

Skobridis, K., 469

Smet, M., 485

Sobarzo-Sánchez, E., 77, 79, 81, 83, 177

Sofin, M., 379

Song, X.-Y., 93

Soudi, A. A., 33

Souldozi, A., 33
Starikova, Z. A., 33

Steinborn, D., 115

Stępniak, K., 37

Steyer, S., 423

Strähle, J., 547, 549

Strasdeit, H., 551

Subramanian, L. R., 547, 549

Sun, F., 535

Sun, J., 221, 328

Sun, L., 499, 501, 503, 506, 509, 513, 515, 517

Sun, T.-H., 341

Sun, X.-J., 243, 247, 249, 305

Sun, Z.-Y., 521

Svoboda, I., 29, 90, 269

Talja, M., 553

Tan, M.-Y., 519

Tanaka, J.-I., 333

Tanaka, T., 26, 27

Tao, X.-T., 543

Taraba, J., 205

Tavani, C., 219

Taylor, D. K., 63, 65, 561

Teijeira, M., 437

Thomas, P., 293

Tiekink, E. R. T., $63,65,67,69,71,73,345$, $347,349,351,354,357,359,361,363$. 365,561

Tinant, B., 59, 145, 194, 475, 477, 572

Tomura, M., 555

Toth, G., 217

Tyagi, A. K., 276

Uriarte, E., 437

Urschel, B., 429

Usman, A., 317

Uytterhoeven, K., 118, 481, 483, 485, 488

Valach, F., 31

Valder, C., 471

Valencia-Matarranz,L., 77, 79, 81, 83, 177

Van Deun, R., 118, 488

Van Langenberg, K. A., 345

Van Meervelt, L., 118, 197, 481, 483, 485, 488

Vanderveer, D., 479

Vértesy, L., 575

Vijayaraghavan, R., 52

Viossat, B, 123

Vocanson, F., 87

Wacker, K., 7

Wagner, Ch., 115

Wang, D.-Q., 243, 247, 249, 251, 253, 255, $257,301,303,305,307,309,311,313$,

$315,319,335$

Wang, G.-P., 245

Wang, P., 25

Wang, R.-J., 535

Wang, S., 491

Wang, X.-J., 243, 247, 249, 253, 301,303, 305,309

Wang, X.-Q., 389

Wang, X.-T., 148

Wang, Y.-F., 255, 257, 311

Wang, Z.-D., 321

Wang, Z.-M., 389

Wartchow, R., 373

Watkin, D. J., 31

Weber, E., 203, 469

Wei, D.-Y., 23, 277

Weidenbruch, M., 101

Weil, M., 155

Wejroch, K., 331

Welter, R., 423

Wendschuh, M., 391, 393, 395

Wilhelm, M., 551 
Wontcheu, J., 285

Wu, C.-J., 97, 157, 297

Wu, Q.-S., 111

Wu, W.-S., 525, 527, 529

Wurst, K., 181, 184, 187

Wuzik, A., 209

Xia, D.-S., 311, 313, 315

Xia, J., 247

Xiao, H.-P., 367, 491, 565

Xie, H.-Z., 111

Xie, L.-M., 245

Xie, Q.-X., 289, 291

Xiong, J., 565

Xu, D., 389

Xu, F.-Y., 535

Xu, W., 25, 325

Xue, G., 323, 325, 389, 545

Yağbasan, R., 175

Yamashita, Y., 555

Yang, F., 321

Yang, S., 499, 501, 503, 506, 509,511,513, $515,517,523$
Yanovsky, A., 33

Yoshimura, M., 1

You, Z.-L., 519

Yu, J.-L., 328

Yu, K.-B., 41, 43, 45, 443

Yu, Q., 251, 307

Yu, S.-Y., 443

Yu, W.-T., 295, 323, 325, 389, 543, 545

Yuan, D.-R., 389

Yuan, J.-X., 93

Yuan, P., 535

Zabel, M., 125

Zeng, Q.-F., 311, 313, 315

Zhang, B., 521

Zhang, B.-S., 231, 421

Zhang, F. X., 26, 27

Zhang, F.-S., 535

Zhang, H., 17, 271, 515

Zhang, M., 521

Zhang, Q.-F., 339

Zhang, R.-Q., 341

Zhang, S.-Y., 85
Zhang, X.-M., 251, 307, 309

Zhao, G.-L., 239

Zhao, J.-T., 17, 165, 167, 169, 171, 271, 273

Zhao, M.-G., 157, 295

Zheng, C., 11, 148

Zheng, Y.-Q., 23, 25, 111, 221, 223, 225,

$227,229,231,277,421$

Zherebtsov, D. A., 163

Zhou, X.-H., 535

Zhou, Y.-L., 563

Zhu, H.-L., 41, 43, 243, 247, 249, 251, 253, $255,257,259,261,263,301,303,305$, $307,309,311,313,315,317,319,321$, $335,499,501,503,506,509,511,513$, $515,517,519,521,523$

Zhu, L.-G., 85, 245, 563

Zhu, N.-W., 341

Zhu, X.-L., 313, 315, 335

Ziegler, T., 547, 549

Zou, Y.-Q., 45, 211, 213,445, 448, 451, 453 